\title{
Maximum Likelihood Angle-Range Estimation for Monostatic FDA-MIMO Radar with Extended Range Ambiguity Using Subarrays
}

\author{
Kaikai Yang, Sheng Hong (D, Qi Zhu, and Yanheng Ye \\ School of Information Engineering, Nanchang University, Nanchang 330031, China \\ Correspondence should be addressed to Sheng Hong; shenghong@ncu.edu.cn
}

Received 29 May 2020; Revised 31 July 2020; Accepted 17 August 2020; Published 8 September 2020

Guest Editor: Qing Shen

Copyright (c) 2020 Kaikai Yang et al. This is an open access article distributed under the Creative Commons Attribution License, which permits unrestricted use, distribution, and reproduction in any medium, provided the original work is properly cited.

\begin{abstract}
In this paper, we consider the joint angle-range estimation in monostatic FDA-MIMO radar. The transmit subarrays are first utilized to expand the range ambiguity, and the maximum likelihood estimation (MLE) algorithm is first proposed to improve the estimation performance. The range ambiguity is a serious problem in monostatic FDA-MIMO radar, which can reduce the detection range of targets. To extend the unambiguous range, we propose to divide the transmitting array into subarrays. Then, within the unambiguous range, the maximum likelihood (ML) algorithm is proposed to estimate the angle and range with high accuracy and high resolution. In the ML algorithm, the joint angle-range estimation problem becomes a high-dimensional search problem; thus, it is computationally expensive. To reduce the computation load, the alternating projection ML (AP-ML) algorithm is proposed by transforming the high-dimensional search into a series of one-dimensional search iteratively. With the proposed AP-ML algorithm, the angle and range are automatically paired. Simulation results show that transmitting subarray can extend the range ambiguity of monostatic FDA-MIMO radar and obtain a lower cramer-rao low bound (CRLB) for range estimation. Moreover, the proposed AP-ML algorithm is superior over the traditional estimation algorithms in terms of the estimation accuracy and resolution.
\end{abstract}

\section{Introduction}

The frequency diverse array (FDA) concept was first proposed at the 2006 IEEE Radar Conference and has received widespread attention because of its range-dependent beam pattern [1-3]. The FDA range-dependent beam pattern is generated by the different carrier frequencies of the array elements; that is, there is a frequency increment between the FDA array element carrier frequency, and the frequency increment is much smaller than the reference array element carrier frequency. Compared with traditional phased array, the FDA has obvious advantages in applications such as beamforming [4], target detection and positioning [5], deceptive jamming suppression [6], and secure communication [7].

The multiple input multiple output (MIMO) radar has excellent spatial resolution and target detection performance
$[8,9]$. Combining FDA and MIMO radar can increase FDA degrees-of-freedom (DOFs). There are two types of FDAMIMO radar, which are the bistatic FDA-MIMO radar and the monostatic FDA-MIMO radar. The bistatic FDA-MIMO radar has the serious problem of angle-range coupling, which increases the difficulty of joint angle-range estimation [10]. However, the angle and range parameters are decoupled in the monostatic FDA-MIMO radar, which facilitates the joint angle-range estimation [11].

Although the angle and range parameters are decoupled in the monostatic FDA-MIMO radar, there exists another serious problem of range ambiguity. The problem of unambiguous range in the monostatic FDA-MIMO radar was pointed in [12], where the maximum unambiguous range was derived. In monostatic FDA-MIMO radar, only within a limited range, the range of target can be estimated without ambiguity, and the unambiguous range is inversely 
proportional to the frequency increment. To our best knowledge, there is only one work discussing how to extend the unambiguous range of monostatic FDA-MIMO radar at present. Inspired by [5], reference [13] proposed doublepulses method to extend the unambiguous range, which is to transmit double-pulses with different frequency increments at different times. However, transmitting double-pulses at different times will reduce the real-time performance of the radar system. Inspired by the method of angle and range decoupled using subarrays in traditional FDA (uniform linear array and uniform frequency increment) radar [14] and bistatic FDA-MIMO radar [10], we propose to extend the range ambiguity for the monostatic FDA-MIMO radar using subarrays. In this method, the FDA transmitting array is uniformly divided into subarrays with different frequency increments. Compared with the double-pulse method, the proposed method can reduce the time cost of the monostatic FDA-MIMO radar system. Meanwhile, in traditional monostatic FDA-MIMO radar, reducing the frequency increment can extend the unambiguous range. However, reducing the frequency increment, the cramer-rao low bound (CRLB) of the range estimation will increase accordingly. The proposed transmitting subarrays can solve this contradictory problem, and a lower-range estimation CRLB while extending the unambiguous range can be obtained.

In addition, the joint angle and range estimation with unambiguous range in monostatic FDA-MIMO radars has received a lot of attention. In [15], the angle and range estimation CRLB, MSE, and resolution of monostatic FDAMIMO radar were analysed and compared with traditional MIMO radar, which illustrated the advantages of monostatic FDA-MIMO radar. The multiple signal classification (MUSIC) algorithm was utilized in monostatic FDA-MIMO radar [16], where the resolution threshold for angle and range estimation was analysed. In [17], the estimation of signal parameters via rotational invariance techniques (ESPRIT) algorithm was used to estimate the target angle and range without search. Compared with the searching algorithm (e.g., MUSIC), the calculation cost of ESPRIT algorithm is reduced, but the estimation accuracy is lower than the searching algorithm. In [18], by using the sparseness of the target in the space, the sparse model of the received signal in monostatic FDA-MIMO radar was established. The sparse model was solved using convex optimization, and finally the angle and range were estimated in a single snapshot. In [12], the design of the coprime array was applied to the monostatic FDA-MIMO radar, which further improves the DOFs. At the same time, an angle and range estimation based on Bayesian learning algorithm was proposed. Reconstruction algorithm based on compressed sensing always subject to the grid-mismatch effect and deteriorating the estimation performance [19]. The off-grid angle and range estimation approach for monostatic FDAMIMO radar with single snapshot based on decoupled atomic norm minimization (DANM) is proposed in [20]. And the estimation performance is better than orthogonal matching pursuit (OMP).

In above related works, the classic MUSIC and ESPRIT algorithms are widely used in angle and range estimation for monostatic FDA-MIMO. However, as a classic parameter estimation algorithm, maximum likelihood estimation (MLE) [21] has not been discussed for angle and range estimation in monostatic FDA-MIMO radar. According to the estimation theory, the estimation performance of MLE is better than MUSIC and ESPRIT algorithms, and the estimation result is closer to the parameter estimation CRLB. Considering this paucity, in this paper, we first utilize the MLE algorithm to estimate angle and range jointly in the monostatic FDA-MIMO radar. The log-likelihood function of angle-range estimation is derived, and the maximum likelihood (ML) cost function of the angle and range is obtained. Since the cost function of MLE requires highdimensional search, the computation load is heavy. To improve the computational efficiency, we propose the alternating projection (AP) ML algorithm, which transforms the high-dimensional search into a series of one-dimensional iterative search [22]. In addition, the angle and range are automatically paired during the iteration.

In this paper, the transmitting steering vector of traditional FDA and FDA with subarrays are derived, based on which the signal model of monostatic FDA-MIMO radar with transmitting subarrays is given. Then, the reason and principle of extending unambiguous range by transmitting subarrays are illustrated. The expression for the unambiguous range of the proposed method is derived and compared with the existing method. After extending the range ambiguity, we utilize the MLE algorithm within the unambiguous range to estimate the angle and range jointly in monostatic FDA-MIMO radar. To further reduce the computational load, the AP-MLE is proposed. In the simulation results, the effectiveness of the proposed method to expand the range ambiguity is verified. The CRLB of angle and range is derived. The estimation performance of the proposed AP-ML estimation algorithm is evaluated, which confirms the superiority of the proposed method in the monostatic FDA-MIMO radar with subarrays.

The main contributions of this paper are summarized as follows:

(i) The method to extend the range ambiguity by transmitting subarrays is proposed in the monostatic FDA-MIMO radar. Compared with the existing method of decreasing the frequency increment in monostatic FDA-MIMO radar, the proposed method can obtain a lower range estimate CRLB. Compared with the existing double-pulse method, the proposed method can reduce the time cost of the radar system.

(ii) The AP-ML algorithm for joint angle and range estimation in the monostatic FDA-MIMO radar with transmitting subarrays is proposed. The specific algorithm flow is given, and the estimation performance is evaluated. Simulation results show that the estimation accuracy and resolution ability of the proposed AP-MLE algorithm are significantly superior over the classic MUSIC and ESPRIT algorithms. 
Notations: Superscripts $[\cdot]^{T},[\cdot]^{H}$, and $(\cdot)^{-1}$ denote complex transpose, conjugate transpose, and inverse operation, respectively. $\otimes$ denotes Kronecker product. $\mathrm{lcm}(\cdot)$ denotes least common multiple operation. $\propto$ means proportional relationship. trace $(\cdot)$ denotes trace operation. $\odot$ denotes Hadamard product. $\operatorname{Re}(\cdot)$ means taking the real part. $1_{2 \times 2}$ represents $2 \times 2$ matrix filled with ones.

\section{Signal Model}

The frequency diverse array is different from the traditional array in that there is a nonzero frequency increment $\Delta f$ in the carrier frequency between its array elements, and this frequency increment $\Delta f$ is much smaller than the carrier frequency of the reference array element. The carrier frequency of the $m$-th array element is

$$
f_{m}=f_{1}+(m-1) \Delta f, \quad m=1,2, \ldots, M,
$$

where $f_{1}$ represents the reference array element carrier frequency, and $M$ represents the number of array elements.

In this paper, we consider a monostatic FDA-MIMO radar system with transmitting subarrays. The transmitting subarray monostatic FDA-MIMO radar system is shown in Figure 1 . The transmitting array is uniformly divided into $K$ subarrays, where each subarray is a ULA with $M$ array elements, the spacing between subarrays element and subarrays is $d_{t}$. Assuming the frequency increment of the $k$-th subarray is $\Delta f_{k}$. Receiving array is a ULA with $N$ array elements, and the spacing between receiving array elements is $d_{r}$.

Suppose there is a target at the far field of the monostatic FDA-MIMO radar. The angle of the target relative to the array is $\theta$, and the two-way propagation range of the target is $r$. In this case, the transmitting steering vector of transmitting array is $[10]$

$$
\mathbf{a}_{t}(\theta, r)=\left[\mathbf{a}_{t 1}(\theta, r)^{T}, \ldots, \mathbf{a}_{t k}(\theta, r)^{T}, \ldots, \mathbf{a}_{t K}(\theta, r)^{T}\right]^{T},
$$

where $\mathbf{a}_{t k}(\theta, r)$ is the transmitting steering vector of the $k$-th subarray and $\mathbf{a}_{t k}(\theta, r)$ can be expressed as [1-3]

$$
\mathbf{a}_{t k}(\theta, r)=e^{-j(2 \pi / c)\left((k-1) M d_{t} f_{1} \sin (\theta)-\left(f_{k, 1}-f_{1,1}\right) r\right)}\left[1, \ldots, e^{-j(M-1) \Delta \varphi_{k}(\theta, r)}\right]^{T},
$$

where $\Delta \varphi_{k}(\theta, r)=(2 \pi / c)\left(d_{t} f_{1} \sin (\theta)-\Delta f_{k} r\right)$ and $c$ is speed of light. $f_{k, 1}$ represents the carrier frequency of the first element of the $k$-th subarray, and definition $f_{k, m}$ represents the carrier frequency of the $m$-th element of the $k$ th subarray, which can be written as

$$
f_{k, m}=f_{k, 1}+(m-1) \Delta f_{k}, \quad m=1,2, \ldots, M .
$$

If $K=1$ or $\Delta f_{1}=\cdots=\Delta f_{k}=\cdots=\Delta f_{K}$ and $f_{k, 1}=$ $f_{k-1,1}+M \Delta f_{k-1}$, the transmitting subarrays degenerates into nontransmitting subarrays (transmitting frequency diverse array with ULA and uniform frequency increment).

Assuming the transmitting subarrays transmit $\mathrm{KM}$ orthogonal signals, the narrow-band complex signal transmitted by the $m$-th element in the $k$-th subarray can be expressed as

$$
s_{k, m}(t)=\phi_{k, m}(t) e^{j 2 \pi f_{k, m} t},
$$

where $\phi_{k, m}(t)$ represents the $k m$-th orthogonal signal.

Suppose there are $P$ static and irrelevant targets in the far field. The transmitting subarrays transmit $K M$ signals, after the target is reflected, the signal is received and sampled by the receiving array, after matched filtering, the received signal can be written as $[10,20]$

$$
\mathbf{x}(l)=\sum_{p=1}^{P} \mathbf{a}_{r}\left(\theta_{p}\right) \otimes \mathbf{a}_{t}\left(\theta_{p}, r_{p}\right) \beta_{p}(l)+\mathbf{n}(l), \quad l=1, \ldots, L,
$$

where $L$ is snapshots of the received signal, $\theta_{p}$ indicates the angle (direction of arrival (DOA) and direction of departure (DOD)) of the $p$-th target, and $r_{p}$ indicates the two-way range for the $p$-th target. $\beta_{p}(l)$ represents the complex amplitude of the received $p$-th target, and $\mathbf{a}_{r}\left(\theta_{p}\right)=\left[1, \ldots, e^{j(2 \pi / c)(N-1) d_{r} f_{1} \sin \left(\theta_{p}\right)}\right]^{T}$ is the receiving array steering vector. $\mathbf{n}(l)$ is the $M N$-dimensional complex Gaussian noise vector with zero mean and variance of $\sigma^{2}$.

Equation (6) can be written as

$$
\mathbf{x}(l)=\mathbf{A} \boldsymbol{\beta}(l)+\mathbf{n}(l), \quad l=1, \ldots, L,
$$

where $\mathbf{A}=\left[\mathbf{a}_{r}\left(\theta_{1}\right) \otimes \mathbf{a}_{t}\left(\theta_{1}, r_{1}\right), \ldots, \mathbf{a}_{r}\left(\theta_{P}\right) \otimes \mathbf{a}_{t}\left(\theta_{P}, r_{P}\right)\right] \in \mathbb{C}$ $K N M \times P$ is the array manifold matrix, $\beta(l)=\left[\beta_{1}(l), \ldots, \beta_{p}\right.$ $\left.(l), \ldots, \beta_{P}(l)\right]^{T} \in \mathbb{C}^{P \times 1}$ is the complex amplitude vector of $P$ targets.

\section{Extend the Range Ambiguity Using Subarrays}

In traditional radar system, ambiguity range is caused by range-folded echo due to pulse repetition frequency (PRF). However, in FDA-MIMO radar, the angle-range of the target can be estimated jointly. Similarly, the range ambiguity problem also exists in FDA-MIMO radar. The range ambiguity in FDA-MIMO radar is caused by the multiple peak values in range dimension of the angle-range beamforming [13]. In this section, we explain the range ambiguity problem from the angle-range estimation principle. In this case, the problem of the multiple peak values in range dimension of the angle-range beamforming turns into the multiple peak values in range dimension of the angle-range spectrum. In addition, we use method of subarrays to extend the range between the multiple peaks and it is equivalent to extending unambiguous range in FDA-MIMO radar.

It can be seen from equation (7) that the angle and range in monostatic FDA-MIMO are decoupled. The angle of the target can be estimated separately from the receiving steering vector. Subsequently, the range of the target can be estimated from the transmitting steering vector. When estimating the angle and range of $p$-th target for transmitting frequency diverse array with ULA and uniform frequency increment, it is equivalent to estimating the phase difference $\Delta \varphi\left(\theta_{p}, r_{p}\right)=(2 \pi / c)\left(d_{t} f_{1} \sin \theta_{p}-\Delta f r_{p}\right) \quad$ in the 


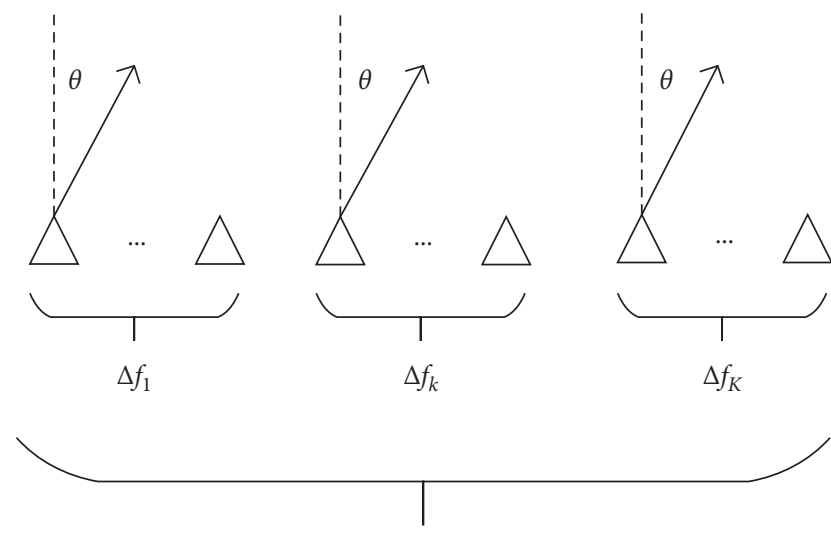

(a)

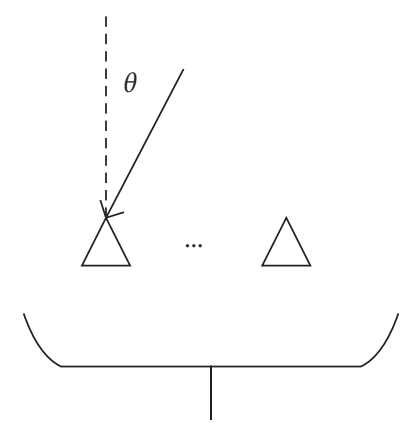

(b)

Figure 1: Transmitting subarrays monostatic FDA-MIMO radar system. (a) Transmitting array. (b) Receiving array.

transmitting steering vector and the phase difference $\Delta \varphi\left(\theta_{p}\right)=(2 \pi / c)\left(d_{r} f_{1} \sin \theta_{p}\right)$ in the receiving steering vector. Obviously, the angle $\theta_{p}$ of $p$-th target can be estimated from the receiving steering vector without unambiguous. The range $r_{p}$ of $p$-th target can be estimated from the transmitting steering vector. However, the phase difference $\Delta \varphi\left(\theta_{p}, r_{p}\right)$ is ambiguous with range.

We suppose the estimated value $\phi$ for the phase difference $\Delta \varphi\left(\theta_{p}, r_{p}\right)$ by the estimation algorithms is in the range of $[-\pi, \pi)$, i.e., $\phi \in[-\pi, \pi)$. The range of the $p$-th target can be retrieved from the equation $\Delta \varphi\left(\theta_{p}, r_{p}\right)=\phi$, $\phi \in[-\pi, \pi)$. We assume that the truth value of the $p$-th target range is $r_{t}$ and the truth value of the $p$-th target angle is $\theta_{t}$. Then, we have

$$
\left(\frac{2 \pi}{c}\right)\left(d_{t} f_{1} \sin \theta_{t}-\Delta f\left(r_{t}+q r_{\mathrm{amb}}\right)\right)=\phi, \quad q=0, \pm 1, \pm 2, \ldots,
$$

where $r_{\mathrm{amb}}$ is the ambiguity range. From (8), we can see that $r_{\mathrm{amb}}=c / \Delta f$, which means that the range ambiguity is determined by the frequency increment $\Delta f$. We can also conclude that there is a periodic ambiguity in the estimated range and the periodic range is $c / \Delta f$. That means the maximum unambiguous detection range of monostatic FDA-MIMO radar is $c / \Delta f$ and the maximum unambiguous range is inversely proportional to the frequency increment. Hence, we propose the subarrays method to handle the ambiguity range and extend the maximum unambiguous range.

In this paper, we utilize the method of transmitting subarrays to extend the maximum unambiguous range for monostatic FDA-MIMO radar. In the transmitting subarrays, the frequency increment of the $k$-th subarray is $\Delta f_{k}$. When range of the target estimated from the transmitter with transmitting subarrays, it is equivalent to estimating the phase difference $\Delta \varphi_{k}\left(\theta_{p}, r_{p}\right)=(2 \pi / c)\left(d_{t} f_{1} \sin \theta_{p}-\Delta f_{k} r_{p}\right)$ in the transmitting steering vector and angle of the $p$-th target estimated from receiving steering vector before. We suppose the estimated value $\phi_{k}$ for the phase difference $\Delta \varphi_{k}\left(\theta_{p}, r_{p}\right)$ of $k$-th subarray by the estimation algorithms is in the range of $[-\pi, \pi)$, i.e., $\phi_{k} \in[-\pi, \pi)$. In this case, the ambiguous rule of transmitting subarrays is determined by

$$
\left\{\begin{array}{l}
\left(\frac{2 \pi}{c}\right)\left(d_{t} f_{1} \sin \theta_{t}-\Delta f_{1}\left(r_{t}+q_{1} r_{\mathrm{amb}}^{1}\right)\right)=\phi_{1}, q_{1}=0, \pm 1, \pm 2, \ldots \\
\vdots \\
\left(\frac{2 \pi}{c}\right)\left(d_{t} f_{1} \sin \theta_{t}-\Delta f_{k}\left(r_{t}+q_{k} r_{\mathrm{amb}}^{k}\right)\right)=\phi_{k}, q_{k}=0, \pm 1, \pm 2, \ldots \\
\vdots \\
\left(\frac{2 \pi}{c}\right)\left(d_{t} f_{1} \sin \theta_{t}-\Delta f_{K}\left(r_{t}+q_{K} r_{\mathrm{amb}}^{K}\right)\right)=\phi_{K}, q_{K}=0, \pm 1, \pm 2, \ldots
\end{array}\right.
$$

where $r_{\mathrm{amb}}^{k}$ is the maximum unambiguous range of the $k$-th subarray. Similarly, we can conclude that there is a periodic ambiguity in the range estimated by $k$-the subarray and the periodic range is $c / \Delta f_{k}$. From equation (9), we can conclude that the maximum unambiguous range of transmitting subarrays satisfies 


$$
q r_{\mathrm{amb}}^{\max }=q_{1} r_{\mathrm{amb}}^{1}=\cdots=q_{k} r_{\mathrm{amb}}^{k}=\cdots=q_{K} r_{\mathrm{amb}}^{K}, \quad q=0, \pm 1, \pm 2, \ldots,
$$

where $r_{\mathrm{amb}}^{\max }$ is the maximum unambiguous range of transmitting subarrays. Hence, from the equations (9) and (10), the maximum unambiguous range of monostatic FDAMIMO radar with transmitting subarrays is

$$
r_{\mathrm{amb}}^{\max }=\operatorname{lcm}\left(\frac{c}{\Delta f_{1}}, \ldots, \frac{c}{\Delta f_{k}}, \ldots, \frac{c}{\Delta f_{K}}\right) .
$$

From equation (11), if $\left(\left(c / \Delta f_{1}\right), \ldots,\left(c / \Delta f_{k}\right), \ldots\right.$, $\left.\left(c / \Delta f_{K}\right)\right)$ satisfies the coprime relationship, the maximum unambiguous range is $\left(c / \Delta f_{1}\right) \times \cdots \times\left(c / \Delta f_{k}\right)$ $\times \cdots \times\left(c / \Delta f_{K}\right)$. Therefore, a reasonable configuration of the transmitting subarrays can greatly extend the unambiguous range of the monostatic FDA-MIMO radar. On the contrary, if $K=1$ or $\Delta f_{1}=\cdots=\Delta f_{k}=\cdots=\Delta f_{K} \quad$ and $f_{k, 1}=f_{k-1,1}+M \Delta f_{k-1}$, transmitting subarrays degenerate into nontransmitting subarrays, the unambiguous range will be greatly reduced.

Maximum unambiguous range of traditional, doublepulses, and transmitting subarrays monostatic FDA-MIMO radar is shown in Table 1 . The maximum unambiguous range of traditional monostatic FDA-MIMO radar is $c / \Delta f$. The maximum unambiguous range of transmitting doublepluses monostatic FDA-MIMO radar is $\operatorname{lcm}\left(\left(c / \Delta f_{1}\right),\left(c / \Delta f_{2}\right)\right)$ and the maximum unambiguous range of transmitting subarrays monostatic FDA-MIMO radar is $\operatorname{lcm}\left(\left(c / \Delta f_{1}\right), \ldots,\left(c / \Delta f_{k}\right), \ldots,\left(c / \Delta f_{K}\right)\right)$. Therefore, both the method of double-pulse and transmitting subarrays can extend the maximum unambiguous range. If the method of double-pulse is to obtain the same size of the maximum unambiguous range as the transmitting subarrays, it is necessary to transmit $K$-pulses with different frequency increments at different times, which will greatly increase the time cost of the radar system. Compared with the method of double-pulses, the method of transmitting subarrays can reduce the time cost of the radar system.

\section{The AP-ML Algorithm for Angle- Range Estimation}

4.1. ML Algorithm for Angle-Range Estimation. In this part, the MLE algorithm is used to estimate angle and range for monostatic FDA-MIMO. First, the log-likelihood function of angle and range estimation is derived. Second, through simplification, the ML cost function about angle and range estimation is obtained. Finally, an AP algorithm is used for dimensionality reduction iterative search. In iterative search, angle and range are automatically matched, and no additional angle and range matching algorithm is required.

According to equation (7) array receiving signal model, the parameter to be estimated is $\eta=\left\{\theta^{T}, \mathbf{r}^{T}, \mathbf{Z}, \sigma^{2}\right\}$, where

$$
\begin{aligned}
\boldsymbol{\theta} & =\left[\theta_{1}, \theta_{2}, \ldots, \theta_{P}\right]^{T}, \\
\mathbf{r} & =\left[r_{1}, r_{2}, \ldots, r_{P}\right]^{T},
\end{aligned}
$$

$$
\mathbf{Z}=\left[\boldsymbol{\beta}(1)^{T}, \ldots, \boldsymbol{\beta}(l)^{T}, \ldots, \boldsymbol{\beta}(L)^{T}\right] .
$$

Under the complex Gaussian noise with zero mean variance of $\sigma^{2}$, suppose the noises are independent of each other, the noise and signal also satisfy the independent relationship. At this time, the array received signal obeys $\mathbf{X} \sim N_{c}\left(\mathbf{A S}, \sigma^{2} \mathbf{I}_{\mathrm{KMN}}\right)$ [23], and the joint probability density function (PDF) of $\mathbf{X}$ can be represented as

$$
f_{X}(\mathbf{X} ; \boldsymbol{\eta})=\prod_{l=1}^{L} \frac{1}{\left(2 \pi \sigma^{2}\right)^{K M N / 2}} \exp \left[-\frac{(\mathbf{x}(l)-\mathbf{A} \boldsymbol{\beta}(l))^{H}(\mathbf{x}(l)-\mathbf{A} \boldsymbol{\beta}(l))}{2 \sigma^{2}}\right],
$$

where $\mathbf{X}=[\mathbf{x}(1), \ldots, \mathbf{x}(l), \ldots, \mathbf{x}(L)]$.

By performing the logarithm operation on the joint PDF, the log-likelihood function is obtained as

$$
\begin{aligned}
L(\boldsymbol{\eta})= & \ln \left\{f_{X}(\mathbf{X} ; \boldsymbol{\eta})\right\}=-\frac{\mathrm{KMNL}}{2} \ln \left(2 \pi \sigma^{2}\right) \\
& -\frac{1}{2 \sigma^{2}} \sum_{l=1}^{L}(\mathbf{x}(l)-\mathbf{A} \boldsymbol{\beta}(l))^{H}(\mathbf{x}(l)-\mathbf{A} \boldsymbol{\beta}(l)) .
\end{aligned}
$$

By taking the derivative of $\sigma^{2}$ setting the derivative function to zero, we can obtain the maximum likelihood estimation of $\sigma^{2}$ as

$$
\widehat{\sigma}^{2}=\frac{1}{\operatorname{KMNL}} \sum_{l=1}^{L}(\mathbf{x}(l)-\mathbf{A} \boldsymbol{\beta}(l))^{H}(\mathbf{x}(l)-\mathbf{A} \boldsymbol{\beta}(l)) .
$$

Substituting $\widehat{\sigma}^{2}$ into equation (13), the new log-likelihood function as

$$
\begin{aligned}
L(\boldsymbol{\theta}, \mathbf{r}, \mathbf{Z})= & -\frac{\mathrm{KMNL}}{2}-\frac{\mathrm{KMNL}}{2} \\
& \cdot \ln \left(\frac{2 \pi}{\mathrm{KMNL}} \sum_{l=1}^{L}(\mathbf{x}(l)-\mathbf{A} \boldsymbol{\beta}(l))^{H}(\mathbf{x}(l)-\mathbf{A} \boldsymbol{\beta}(l))\right) .
\end{aligned}
$$

From equation (16), the new log-likelihood function satisfies the following relationship:

$$
L(\boldsymbol{\theta}, \mathbf{r}, \mathbf{Z}) \propto-\sum_{l=1}^{L}(\mathbf{x}(l)-\mathbf{A} \boldsymbol{\beta}(l))^{H}(\mathbf{x}(l)-\mathbf{A} \boldsymbol{\beta}(l)) .
$$

Then, the maximum likelihood estimation of angle and range becomes

$$
(\widehat{\boldsymbol{\theta}}, \widehat{\mathbf{r}}, \widehat{\mathbf{Z}})_{\mathrm{ML}}=\arg \max _{\theta, r, Z}(L(\boldsymbol{\theta}, \mathbf{r}, \mathbf{Z})) .
$$

Because $\mathbf{x}(l)=\mathbf{A} \boldsymbol{\beta}(l)+\mathbf{n}(l)$ is a linear model about $\beta(l)$, we can know from the best likelihood estimation theorem of the linear model

$$
\widehat{\boldsymbol{\beta}}(l)_{\mathrm{ML}}=\left(\mathbf{A}^{H} \mathbf{A}\right)^{-1} \mathbf{A}^{H} \mathbf{x}(l) .
$$

Taking equations (19) into (18) and simplifying, the maximum likelihood cost function for angle and range estimation can be described as 
TABLE 1: Maximum unambiguous range of traditional, double-pulses, and transmitting subarrays monostatic FDA-MIMO radar.

\begin{tabular}{lccc}
\hline FDA & Traditional & Double-pulses & Transmitting subarrays \\
\hline Maximum unambiguous range $(\mathrm{m})$ & $c / \Delta f$ & $\operatorname{lcm}\left(\left(c / \Delta f_{1}\right),\left(c / \Delta f_{2}\right)\right)$ & $\operatorname{lcm}\left(\left(c / \Delta f_{1}\right), \ldots,\left(c / \Delta f_{k}\right), \ldots,\left(c / \Delta f_{K}\right)\right)$ \\
\hline
\end{tabular}

$$
(\widehat{\boldsymbol{\theta}}, \widehat{\mathbf{r}})_{\mathrm{ML}}=\arg \max _{\theta, r} \operatorname{trace}\left(\mathbf{P}_{\mathbf{A}} \mathbf{R}\right),
$$

where $\mathbf{P}_{\mathbf{A}}=\mathbf{A}\left(\mathbf{A}^{H} \mathbf{A}\right)^{-1} \mathbf{A}^{H}$ is the projection matrix and $\mathbf{R}=$ $(1 / L) \sum_{l=1}^{L} \mathbf{x}(l) \mathbf{x}^{H}(l)$ is the signal covariance matrix.

ML cost function is a high-dimensional search problem. When the number of targets is $P$, a global search will be conducted in the $2 P$-dimensional space. In order to improve the calculation efficiency, we further simplify the $2 P$-dimensional search into a series of one-dimensional iterative search by AP algorithm.

4.2. AP Iterative Search for ML Cost Function. AP algorithm is a simple and effective method to solve the multidimensional searching problem. It simplifies the multidimensional searching into a series of one-dimensional searching and thus can greatly reduce the computational load. However, if the objective function is not convex, the initial value of the parameter will greatly influence the result of searching. In order to prevent the algorithm from falling into the local optimal value, a good initialization parameter value is very important. The method in [24] is used to initialize angle and range.

Firstly, the first group of angle and range initial values is obtained as follows:

$$
\left(\widehat{\theta}_{1}^{(0)}, \widehat{r}_{1}^{(0)}\right)=\arg \max _{\theta, r} \operatorname{trace}\left\{\mathbf{P}_{\mathbf{a}_{r}(\theta) \otimes \mathbf{a}_{t}(\theta, r)} \mathbf{R}\right\} .
$$
by

After that, the second group of angle and range is solved

$$
\left(\widehat{\theta}_{2}^{(0)}, \widehat{r}_{2}^{(0)}\right)=\arg \max _{\theta, r} \operatorname{trace}\left\{\mathbf{P}_{\left[\mathbf{a}_{r}\left(\hat{\theta}_{1}^{(0)}\right) \otimes \mathbf{a}_{t}\left(\hat{\theta}_{1}^{(0)}, \widehat{r}_{1}^{(0)}\right), \mathbf{a}_{r}(\theta) \otimes \mathbf{a}_{t}(\theta, r)\right]} \mathbf{R}\right\} .
$$

According to the method of equations (21) and (22), initialize the $P$ groups of angle and range in sequence.

Next, iteratively update the $P$ groups of angle and range until the algorithm iteration stop condition is satisfied. In the iterative process, perform a one-dimensional search for angle and range, respectively:

$$
\begin{aligned}
& \hat{\theta}_{p}^{(j+1)}=\arg \max _{\theta} \operatorname{trace}\left\{\mathbf{P}_{\left[\widehat{\mathbf{A}}\left(\Theta^{(j)}\right), \mathbf{a}_{r}(\theta) \otimes \mathbf{a}_{t}\left(\theta, \widehat{r}_{p}^{(j)}\right)\right]} \mathbf{R}\right\}, \\
& \widehat{r}_{p}^{(j+1)}=\arg \max _{r} \operatorname{trace}\left\{\mathbf{P}_{\left[\widehat{\mathbf{A}}\left(\Theta^{(j)}\right), \mathbf{a}_{r}\left(\widehat{\theta}_{p}^{(j)}\right) \otimes \mathbf{a}_{t}\left(\widehat{\theta}_{p}^{(j)}, r\right)\right]} \mathbf{R}\right\},
\end{aligned}
$$

where $\Theta^{(j)}$ represents the $P$-1 pair angle and range from the $j$-th iteration, which can be described as

$$
\Theta^{(j)}=\left[\left(\theta_{1}^{(j)}, r_{1}^{(j)}\right), \ldots,\left(\theta_{p-1}^{(j)}, r_{p-1}^{(j)}\right),\left(\theta_{p+1}^{(j)}, r_{p+1}^{(j)}\right), \ldots,\left(\theta_{P}^{(j)}, r_{P}^{(j)}\right)\right]
$$

Finally, the iteration stop condition is

$$
\begin{array}{r}
\left|\hat{\theta}_{p}^{(j+1)}-\widehat{\theta}_{p}^{(j)}\right| \leq \delta, \\
\left|\widehat{r}_{p}^{(j+1)}-\widehat{r}_{p}^{(j)}\right| \leq \varepsilon,
\end{array}
$$

where $p=1, \ldots, P, \delta$, and $\varepsilon$ are error thresholds. When (26) and (27) are satisfied at the same time or the iteration reaches the maximum number, the iteration stops.

The steps for the angle and range estimation in monostatic FDA-MIMO radar using AP-MLE are described as follows:

Step 1: $j=0$, initialize the angle-range parameters according to equations (21) and (22).

Step $2: j=j+1$, iteratively and update the angle and range parameters according to equations (23) and (24).

Step 3: if (26) and (27) are satisfied, or the maximum number of iterations is reached, the algorithm stops, otherwise repeat Step 2.

\section{Simulation Results}

In this part, we design some experiments to prove the efficiency of the proposed method of transmitting subarrays to extend the unambiguous range for monostatic FDA-MIMO radar and the superiority of the proposed AP-ML angle and range estimation for monostatic FDA-MIMO radar.

5.1. Simulation 1. In order to verify the effectiveness of the proposed transmitting subarrays to extend the unambiguous range for monostatic FDA-MIMO radar, simulation 1 gives the unambiguous range of transmitting subarrays monostatic FDA-MIMO radar and nontransmitting subarrays monostatic FDA-MIMO radar by MUSIC spectrum.

Consider the number of transmitting subarrays is $K=2$, the number of subarrays elements is $M=6$, and the frequency increment $\Delta f_{1}$ of the first subarray is $2 \mathrm{kHz}$. The frequency increment $\Delta f_{2}$ of the second subarray is $5 \mathrm{kHz}$. The carrier frequency of the first subarray reference array element is $10 \mathrm{GHz}$, and the carrier frequency of the second subarray reference array element is $10.000012 \mathrm{GHz}$. The number of array elements of the receiving array is $N=8$, and $d_{t}=d_{r}=\left(c / 2 f_{\mathrm{KM}}\right)$. Suppose a target is located at $\left(20^{\circ}\right.$, $50 \mathrm{~km})$, under the conditions of signal-to-noise ratio $(\mathrm{SNR})=20 \mathrm{~dB}$, and the number of snapshots $L$ is 100 , the following gives the monostatic FDA-MIMO MUSIC spectrum with frequency increment $\Delta f_{1}=\Delta f_{2}=2 \mathrm{kHz}$, $\Delta f_{1}=\Delta f_{2}=5 \mathrm{kHz}$, and $\Delta f_{1}=2 \mathrm{kHz}, \Delta f_{2}=5 \mathrm{kHz}$. 
Figure 2 shows the MUSIC spectrum with $\Delta f_{1}=\Delta f_{2}=2 \mathrm{kHz}$; in this case, the unambiguous range of nontransmitting subarrays (traditional FDA) is $150 \mathrm{~km}$. Figure 3 shows the MUSIC spectrum with $\Delta f_{1}=\Delta f_{2}=5 \mathrm{kHz}$; in this case, the unambiguous range of nontransmitting subarrays is $60 \mathrm{~km}$. Figure 4 shows the MUSIC spectrum with $\Delta f_{1}=2 \mathrm{kHz}$ and $\Delta f_{2}=5 \mathrm{kHz}$; in this case, the unambiguous range of transmitting subarrays is $300 \mathrm{~km}$. Comparing Figures 2 and 3, under the traditional FDA, the unambiguous range is inversely proportional to the frequency increment. Comparing Figures 2-4, it can be concluded that the proposed transmitting subarrays can extend the unambiguous range for monostatic FDA-MIMO radar.

5.2. Simulation 2. In order to verify the correctness of the proposed AP-ML angle and range estimation for transmitting subarrays monostatic FDA-MIMO radar, simulation 2 gives the estimation result of AP-MLE.

Suppose three targets located at $\left(-10^{\circ}, 151 \mathrm{~km}\right),\left(5^{\circ}\right.$, $152 \mathrm{~km})$, and $\left(30^{\circ}, 154 \mathrm{~km}\right)$, and transmitting subarrays $K=2$, the number of each subarray elements $M=6$, $\Delta f_{1}=2 \mathrm{kHz}$, and $\Delta f_{2}=5 \mathrm{kHz}$. Obviously three targets are within the unambiguous range. In the case of $\mathrm{SNR}=10 \mathrm{~dB}$ and snapshot number $L=100,100$ Monte Carlo simulation results are given in Figure 5. The angle search step is $0.05^{\circ}$, and the range search step is $5 \mathrm{~m}$.

Figure 5 shows the angle and range estimation results of AP-MLE within the unambiguous range. From Figure 5, we can know the AP-MLE algorithm can complete the angle and range estimation for transmitting subarrays monostatic FDA-MIMO radar. At SNR $=10 \mathrm{~dB}, 100$ estimations are closer to the real angle and range. In addition, in the AP iteration, the 3 groups of angle and range are automatically paired without additional pairing algorithm.

5.3. Simulation 3. Simulation 3 uses the average number of iterations curves versus SNR to represent the efficiency of the AP-ML angle and range estimation for transmitting subarrays monostatic FDA-MIMO radar. The simulation conditions are the same as for simulation 2 .

Figure 6 gives the average number of iterations curves versus SNR of AP-ML angle and range estimation for transmitting subarrays monostatic FDA-MIMO radar. As the SNR increases, the number of iterations required decreases. When SNR is greater than or equal to $20 \mathrm{~dB}$, APMLE only needs about two iterations and the efficiency of the AP-MLE is significant.

5.4. Simulation 4. In order to evaluate the estimation performance of the AP-MLE algorithm, the AP-MLE root mean square error (RMSE) of the angle and range against the SNR is compared with the MUSIC algorithm, ESPRIT algorithm (angle and range pairing algorithm in [25]), paper [13], and angle-range estimation CRLB. The CRLB expression is given by equation (28), the angle and range RMSE expressions are given by equations (35) and (36).

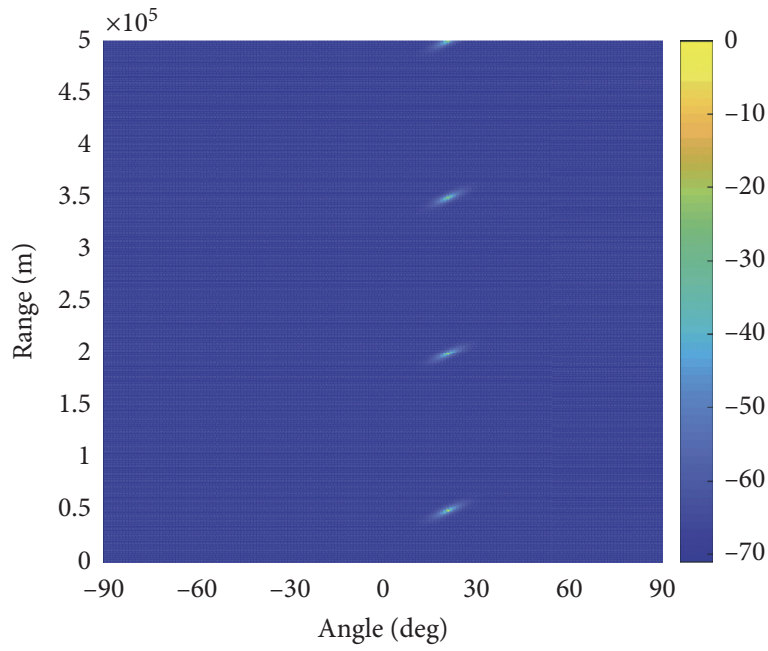

FIgURE 2: MUSIC spectrum with $\Delta f_{1}=\Delta f_{2}=2 \mathrm{kHz}$.

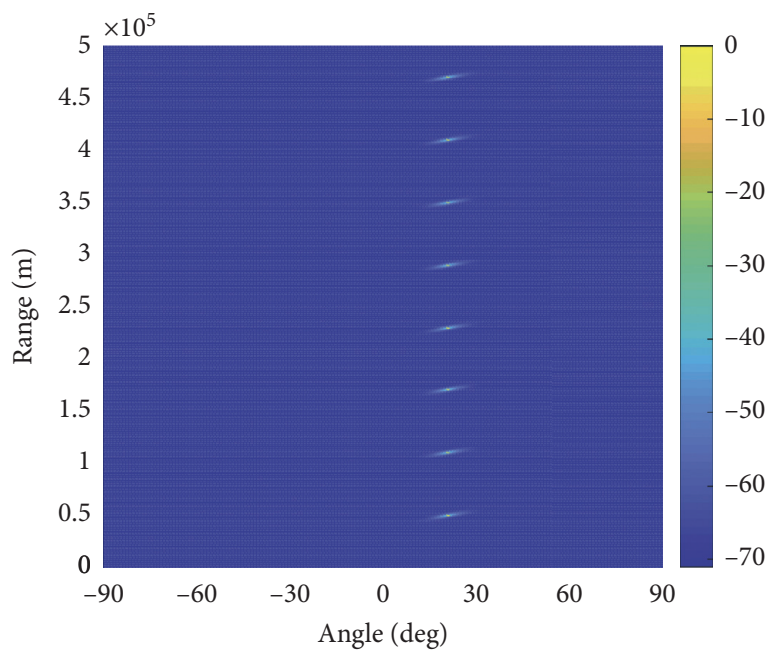

FIgURE 3: MUSIC spectrum with $\Delta f_{1}=\Delta f_{2}=5 \mathrm{kHz}$.

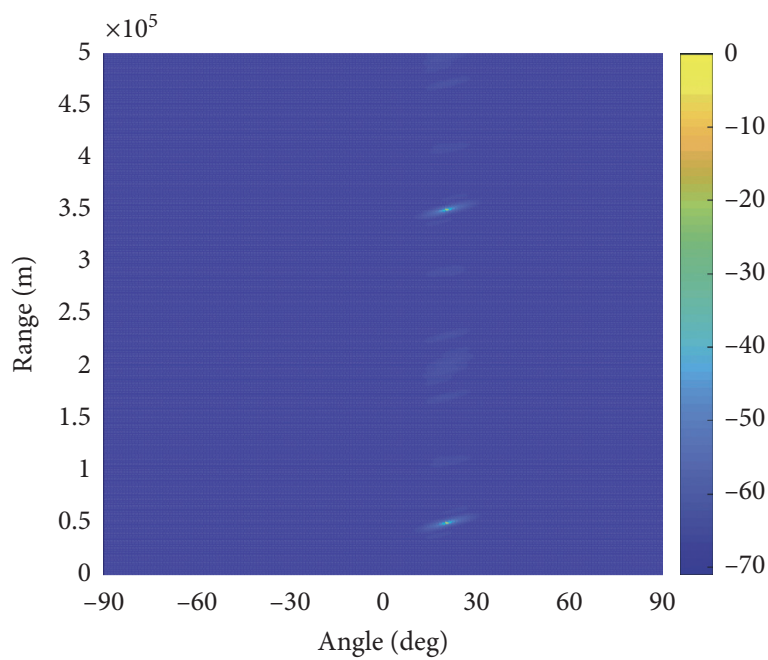

FIgUre 4: MUSIC spectrum with $\Delta f_{1}=2 \mathrm{kHz}$ and $\Delta f_{2}=5 \mathrm{kHz}$. 


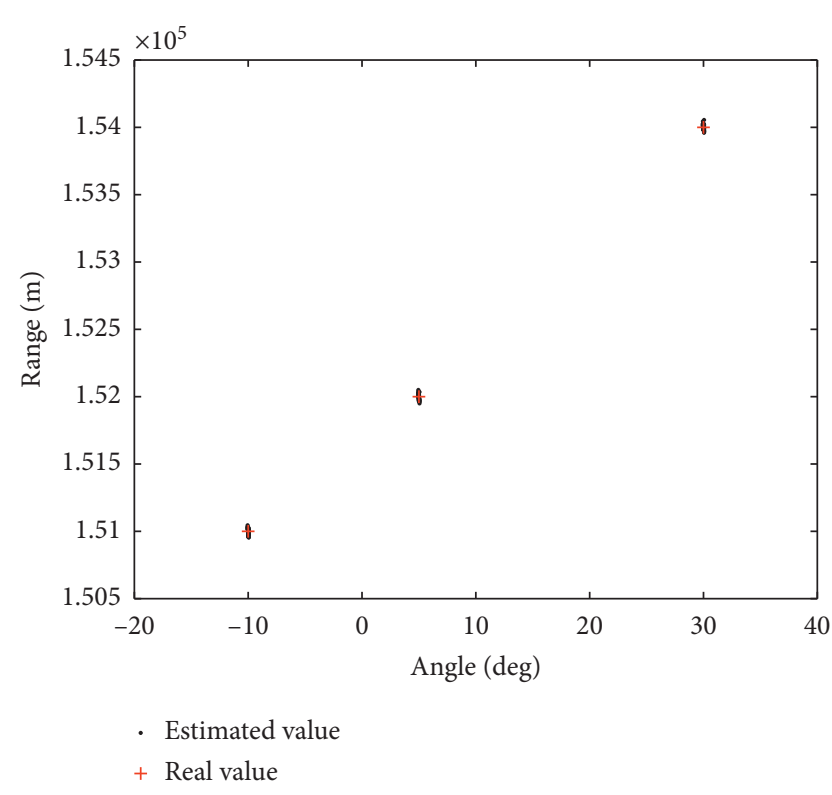

Figure 5: Angle and range estimation results of AP-MLE.

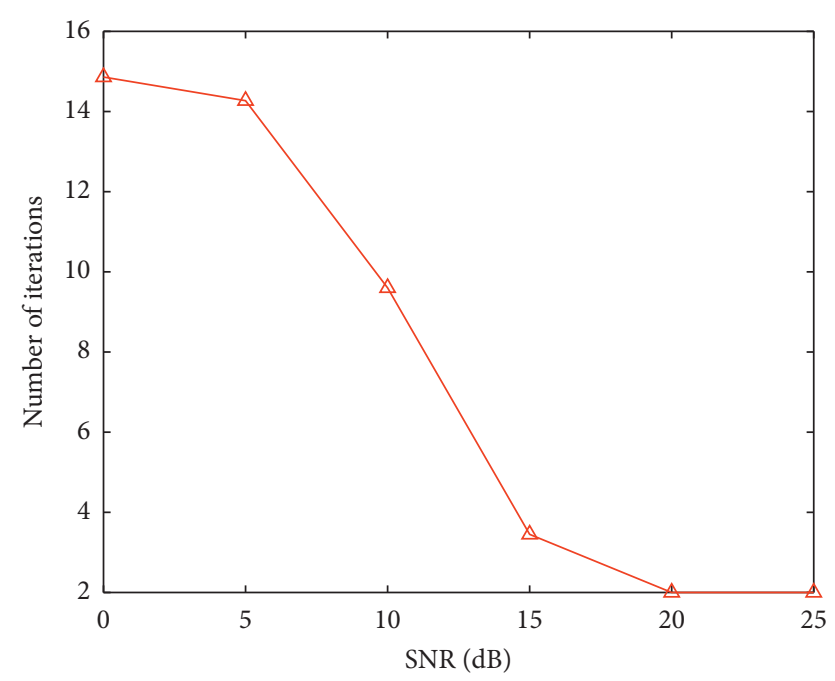

Figure 6: Average number of iterations curves versus SNR.

$$
\operatorname{CRLB}(\boldsymbol{\Theta})=\frac{\sigma^{2}}{2 L}\left\{\operatorname{Re}\left[\left(\mathbf{C}^{H} \mathbf{P}_{\mathbf{A}}^{\perp} \mathbf{C}\right) \odot\left(\mathbf{R}_{\beta} \otimes 1_{2 \times 2}\right)\right]\right\}^{-1},
$$

where

$$
\begin{gathered}
\boldsymbol{\Theta}=\left[\theta_{1}, r_{1}, \ldots, \theta_{p}, r_{p}, \ldots, \theta_{P}, r_{P}\right]^{T} \\
\mathbf{C}=\left[\mathbf{d}\left(\theta_{1}\right), \mathbf{d}\left(r_{1}\right), \ldots, \mathbf{d}\left(\theta_{p}\right), \mathbf{d}\left(r_{p}\right), \ldots, \mathbf{d}\left(\theta_{P}\right), \mathbf{d}\left(r_{P}\right)\right] \\
\mathbf{d}\left(\theta_{p}\right)=\frac{\partial \mathbf{a}_{r}\left(\theta_{P}\right) \otimes \mathbf{a}_{t}\left(\theta_{P}, r_{P}\right)}{\partial \theta_{P}} \\
\mathbf{d}\left(r_{p}\right)=\frac{\partial \mathbf{a}_{r}\left(\theta_{P}\right) \otimes \mathbf{a}_{t}\left(\theta_{P}, r_{P}\right)}{\partial r_{P}}
\end{gathered}
$$

$$
\begin{aligned}
& \mathbf{R}_{\beta}=\frac{1}{L} \sum_{l=1}^{L} \boldsymbol{\beta}(l) \boldsymbol{\beta}^{H}(l), \\
& \mathbf{P}_{\mathbf{A}}^{\perp}=\mathbf{I}-\mathbf{P}_{\mathbf{A}} .
\end{aligned}
$$

From equations (31) and (32), the frequency increments have a significant effect on the CRLB of range estimation and have no significant effect on the CRLB of angle estimation. Therefore, we mainly consider the effect of the frequency increment setting on the CRLB of range estimate in this simulation.

$$
\begin{aligned}
& \operatorname{RMSE}_{\theta}=\frac{1}{P} \sum_{p=1}^{P} \sqrt{\frac{1}{D} \sum_{d=1}^{D}\left(\hat{\theta}_{p}^{d}-\theta_{p}\right)^{2}}, \\
& \operatorname{RMSE}_{r}=\frac{1}{P} \sum_{p=1}^{P} \sqrt{\frac{1}{D} \sum_{d=1}^{D}\left(\hat{r}_{p}^{d}-r_{p}\right)^{2}},
\end{aligned}
$$

where $\widehat{\theta}_{p}^{d}$ is the angle value of the $p$-th target estimated at the $d$-th time, $\widehat{r}_{p}^{d}$ is the range value of the $p$-th target estimated at the $d$-th time, and $D$ is the number of Monte Carlo simulations.

Suppose three targets are located at $\left(-5^{\circ}, 11 \mathrm{~km}\right),\left(10^{\circ}\right.$, $13 \mathrm{~km})$, and $\left(35^{\circ}, 14 \mathrm{~km}\right)$, where the angle search step is $0.01^{\circ}$ and the range search step is $1 \mathrm{~m}$. The number of simulations $D=200$ and other simulation conditions are the same as simulation 2 .

Figure 7 shows angle CRLB and RMSE curves versus SNR and Figure 8 shows range CRLB and RMSE curves versus SNR. From Figures 7 and 8, as the SNR increases, the angle and range RMSE of paper [13], MUSIC, ESPRIT, AP-MLE, and CRLBs decrease continuously. However, the angle and range RMSE of $\mathrm{AP}-\mathrm{MLE}$ is closest to angle and range CRLB. So, the performance of angle and range estimation of AP-MLE is better than paper [13], MUSIC, and ESPRIT, and the ESPRIT is the worst.

Next, according to the range estimation CRLB with $\Delta f_{1}=\Delta f_{2}=2 \mathrm{kHz}, \quad \Delta f_{1}=\Delta f_{2}=3 \mathrm{kHz}, \quad \Delta f_{1}=\Delta f_{2}=$ $5 \mathrm{kHz}$, and $\Delta f_{1}=2 \mathrm{kHz}, \Delta f_{2}=5 \mathrm{kHz}$ in Figure 8 , the nontransmitting subarrays monostatic FDA-MIMO radar range estimation CRLB decreases with increasing frequency. However, from equation (8), the max unambiguous range decreases with increasing frequency. Hence, it is impossible to obtain a low-range CRLB and a large unambiguous range at the same time in nontransmitting subarrays monostatic FDA-MIMO radar. However, lower-range estimation CRLB and large unambiguous range can be obtained at the same time in transmitting subarrays monostatic FDA-MIMO radar. That means the proposed transmitting subarrays can solve the relationship between the low-range estimation CRLB and large unambiguous range contradiction.

5.5. Simulation 5. We measure resolution by the resolution probability of the targets. The expression for the threshold is $\chi=\min \left[\left(\sqrt{\left(\theta_{p 1}-\theta_{p 2}\right)^{2}+\left(r_{p 1}-r_{p 2}\right)^{2}}\right) / 2\right], p_{1}, p_{2}=1, \ldots$, 


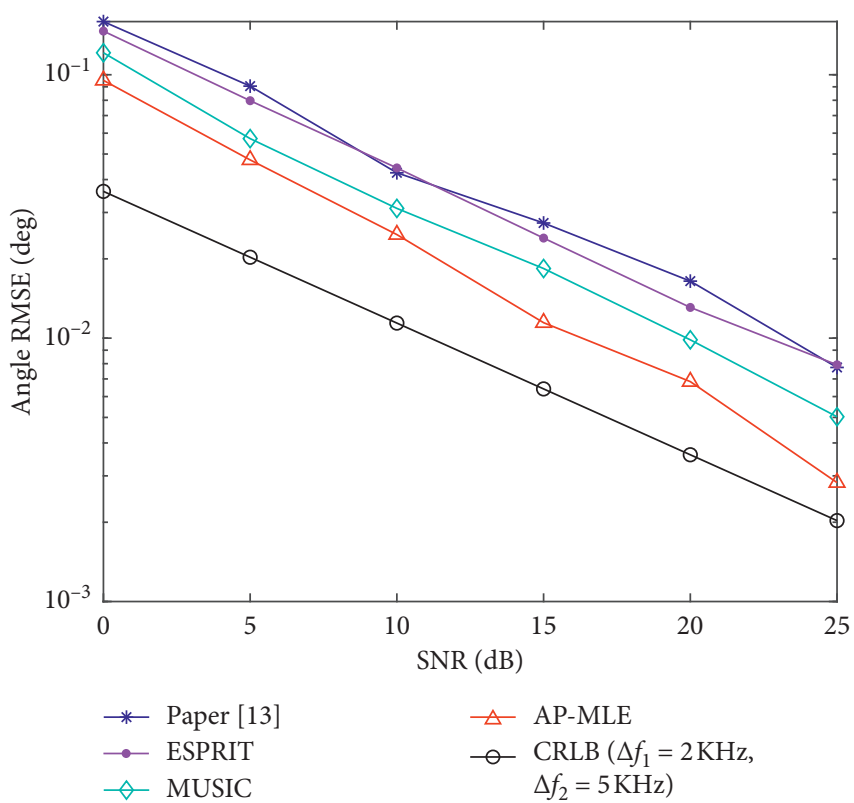

Figure 7: Angle CRLB and RMSE curves versus SNR.

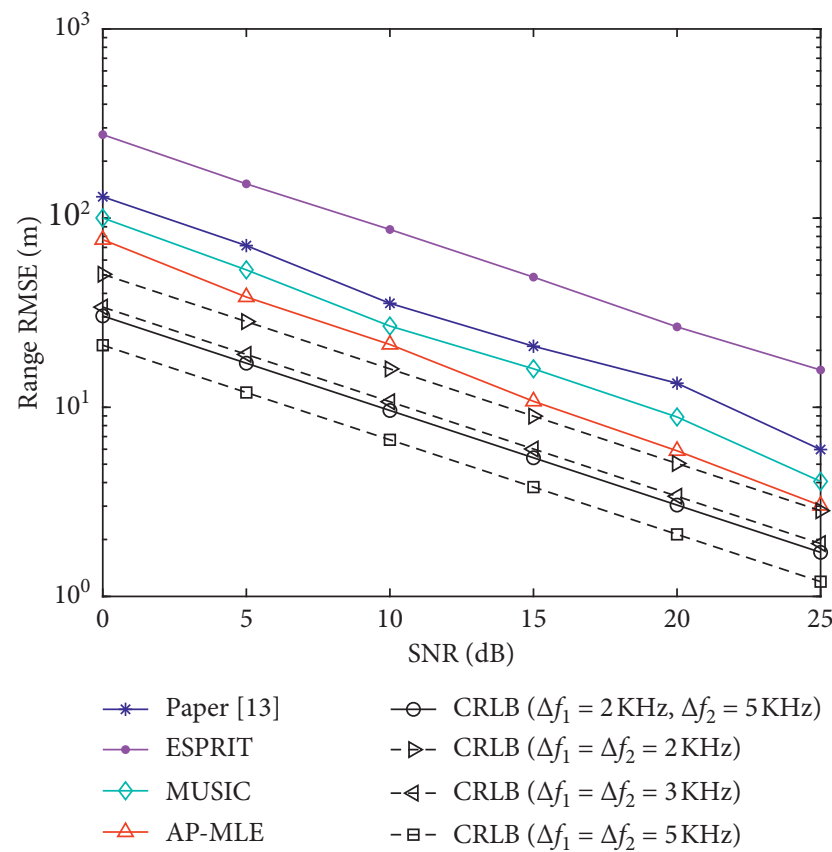

Figure 8: Range CRLB and RMSE curves versus SNR.

$P, p_{1} \neq p_{2}$. If the absolute error of the estimation than $\chi$, the estimation is considered a failure resolution. The estimation angle and range are mapped to the wrong target, which leads to a large error, so that the different targets cannot be distinguished correctly.

Assume that two targets are located at $\left(10^{\circ}, 11 \mathrm{~km}\right)$ and $\left(15^{\circ}, 11.5 \mathrm{~km}\right)$. Other simulation conditions are the same as simulation 2.

Figure 9 gives the resolution probability curves versus SNR. From Figure 9, as the SNR increases, the resolution

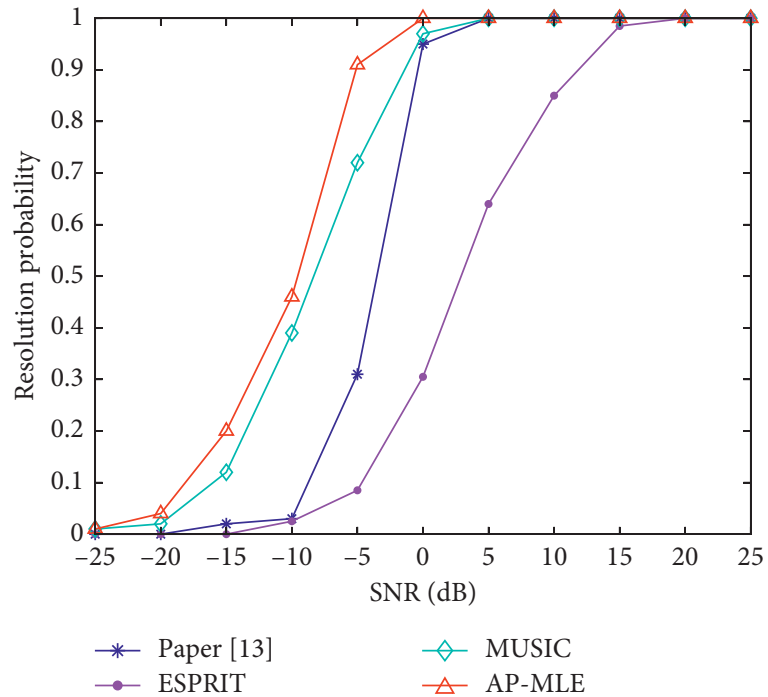

FIGURE 9: Resolution probability curves versus SNR.

probability of AP-MLE reaches 1 at the fastest. From the trend of the curve change, we can conclude the resolution probability of AP-MLE is the best, MUSIC is second, paper [13] is third, and the ESPRIT is the slowest. In addition, the resolution capability of AP-MLE is obviously greater than paper [13], MUSIC, and ESPRIT, and the ESPRIT algorithm.

\section{Conclusions}

This paper proposes to extend the unambiguous range of the monostatic FDA-MIMO radar using the transmitting subarrays. Meanwhile, the proposed transmitting subarrays can solve the relationship between the low range estimation CRLB and large unambiguous range contradiction. In addition, the AP-ML angle and range estimation for monostatic FDA-MIMO radar are proposed. The simulation results prove the superiority of AP-ML angle and range estimation for monostatic FDA-MIMO radar in estimation accuracy and resolution.

\section{Data Availability}

The simulation data used to support the findings of this study are included within the article.

\section{Conflicts of Interest}

The authors declare that there are no conflicts of interest regarding the publication of this paper.

\section{Acknowledgments}

This work was supported by the National Natural Science Foundation of China (61661032), the Young Natural Science Foundation of Jiangxi Province (20181BAB202002), the China Postdoctoral Science Foundation (2017M622102), and the Foundation from China Scholarship Council (201906825071). 


\section{References}

[1] P. Antonik, M. C. Wicks, H. D. Griffiths et al., "Frequency diverse array radars," in Proceedings of the IEEE Conference on Radar, pp. 215-217, IEEE, Verona, NY, USA, April 2006.

[2] A. Basit, W. Khan, S. Khan et al., "Development of frequency diverse array radar technology: a review," IET Radar Sonar \& Navigation, vol. 12, no. 2, pp. 165-175, 2017.

[3] W.-Q. Wang, "Frequency diverse array antenna: new opportunities," IEEE Antennas and Propagation Magazine, vol. 57, no. 2, pp. 145-152, 2015.

[4] W.-Q. Wang, "Range-angle dependent transmit beampattern synthesis for linear frequency diverse arrays," IEEE Transactions on Antennas and Propagation, vol. 61, no. 8, pp. 4073-4081, 2013.

[5] W. Wang and H. Shao, "Range-angle localization of targets by a double-pulse frequency diverse array radar," IEEE Journal of Selected Topics in Signal Processing, vol. 8, no. 1, pp. 106-114, 2014.

[6] J. Xu, G. Liao, S. Zhu, and H. C. So, "Deceptive jamming suppression with frequency diverse MIMO radar," Signal Processing, vol. 113, pp. 9-17, 2015.

[7] J. Lin, Q. Li, J. Yang, H. Shao, and W.-Q. Wang, "Physicallayer security for proximal legitimate user and eavesdropper: a frequency diverse array beamforming approach," IEEE Transactions on Information Forensics and Security, vol. 13, no. 3, pp. 671-684, 2018.

[8] D. W. Bliss and K. W. Forsythe, "Multiple-input multipleoutput (MIMO) radar and imaging: degrees of freedom and resolution," in Proceedings of the Thrity-Seventh Asilomar Conference on Signals, Systems \& Computers, pp. 54-59, Pacific Grove, CA, USA, November 2003.

[9] K. W. Forsythe, D. W. Bliss, and G. S. Fawcett, "Multipleinput multiple-output (MIMO) radar: performance issues," in Proceedings of the Thirty-Eighth Asilomar Conference on Signals, Systems and Computers, pp. 310-315, Pacific Grove, CA, USA, November 2004.

[10] C. Cui, J. Xu, R. Gui, W.-Q. Wang, and W. Wu, "Search-free DOD, DOA and range estimation for bistatic FDA-MIMO radar," IEEE Access, vol. 6, pp. 15431-15445, 2018.

[11] J. Xu, G. Liao, S. Zhu, L. Huang, and H. C. So, "Joint range and angle estimation using MIMO radar with frequency diverse array," IEEE Transactions on Signal Processing, vol. 63, no. 13, pp. 3396-3410, 2015.

[12] S. Qin, Y. D. Zhang, M. G. Amin, and F. Gini, "Frequency diverse coprime arrays with coprime frequency offsets for multitarget localization," IEEE Journal of Selected Topics in Signal Processing, vol. 11, no. 2, pp. 321-335, 2017.

[13] Y. Song, G. Zheng, and G. Hu, "A combined ESPRIT-MUSIC method for FDA-MIMO radar with extended range ambiguity using staggered frequency increment," International Journal of Antennas and Propagation, vol. 2019, Article ID 3056074, 7 pages, 2019.

[14] L. Huang, X. Li, P.-C. Gong, and Z. He, "Frequency diverse array radar for target range-angle estimation," COMPEL - The International Journal for Computation and Mathematics in Electrical and Electronic Engineering, vol. 35, no. 3, pp. 1257-1270, 2016.

[15] J. Xiong, W.-Q. Wang, and K. Gao, "FDA-MIMO radar range-angle estimation: CRLB, MSE, and resolution analysis," IEEE Transactions on Aerospace and Electronic Systems, vol. 54, no. 1, pp. 284-294, 2018.

[16] C. Cui, Y. Yan, W. Wang et al., "Resolution threshold of music algorithm for FDA-MIMO radar," in Proceedings of the 2018
IEEE Radar Conference (RadarConf18), IEEE, Oklahoma City, CA, USA, pp. 0230-0234, April 2018.

[17] Y. Yan, J. Cai, and W. Wang, "Two-stage ESPRIT for unambiguous angle and range estimation in FDA-MIMO radar," Digital Signal Processing, vol. 92, pp. 151-165, 2019.

[18] H. Chen and H. Shao, "Sparse reconstruction based target localization with frequency diverse array MIMO radar," in Proceedings of the 2015 IEEE China Summit and International Conference on Signal and Information Processing (ChinaSIP), pp. 94-98, Chengdu, China, July 2015.

[19] T. Liu, F. Wen, L. Zhang, and K. Wang, "Off-grid DOA estimation for colocated MIMO radar via reduced-complexity sparse Bayesian learning," IEEE Access, vol. 7, pp. 9990799916, 2019.

[20] W. Tang, H. Jiang, and S. Pang, "Gridless angle and range estimation for FDA-MIMO radar based on decoupled atomic norm minimization," in Proceedings of the 2019 IEEE International Conference on Acoustics, Speech and Signal Processing (ICASSP), pp. 4305-4309, IEEE, Brighton, UK, May 2019.

[21] P. Stoica and A. Nehorai, "MUSIC, maximum likelihood, and Cramer-Rao bound: further results and comparisons," IEEE Transactions on Acoustics, Speech, and Signal Processing, vol. 38, no. 12, pp. 2140-2150, 1990.

[22] J. A. Tropp, I. S. Dhillon, R. W. Heath, and T. Strohmer, "Designing structured tight frames via an alternating projection method," IEEE Transactions on Information Theory, vol. 51, no. 1, pp. 188-209, 2005.

[23] I. Bekkerman and J. Tabrikian, "Target detection and localization using MIMO radars and sonars," IEEE Transactions on Signal Processing, vol. 54, no. 10, pp. 3873-3883, 2006.

[24] I. Ziskind and M. Wax, "Maximum likelihood localization of multiple sources by alternating projection," IEEE Transactions on Acoustics, Speech, and Signal Processing, vol. 36, no. 10, pp. 1553-1560, 1988.

[25] C. Duofang, C. Baixiao, and Q. Guodong, "Angle estimation using ESPRIT in MIMO radar," Electronics Letters, vol. 44, no. 12, pp. 770-771, 2008. 